\title{
Saturated electric field effect at semi-insulating GaAs-metal junctions studied with a low energy positron beam
}

\author{
Y. F. Hu, C. C. Ling, C. D. Beling, and S. Fung ${ }^{\text {a) }}$ \\ Department of Physics, The University of Hong Kong, Pokfulam Road, Hong Kong
}

(Received 1 April 1997; accepted for publication 11 July 1997)

\begin{abstract}
The interfacial electric field established under different reverse bias conditions in $\mathrm{Au}$ and $\mathrm{Ni}$ on semi-insulating GaAs junctions has been studied by means of a low energy positron beam. The technique used is that of monitoring the positron drift to the interface through changes in the annihilation radiation lineshape as a function of incident positron beam energy at different reverse biases. The data show a small but clear electric field drift of positrons towards the interface that increases more rapidly at low voltages (less than $50 \mathrm{~V}$ ) which at higher biases tends towards saturation. This confirmation of electric field saturation adds further weight to the picture of an electric field enhanced electron capture cross section for the ionized EL2 defect. Electric field values extracted from the data are compared with results from other techniques and suggest that enhanced electron capture is already occurring at the relatively low built-in fields $\left(\sim 1 \mathrm{kV} \mathrm{cm}{ }^{-1}\right)$ found at the unbiased junction, with a rapid increase of $\mathrm{EL}^{+}$neutralization occurring for biases above $10 \mathrm{~V}$. At still higher fields $\sim 10 \mathrm{kV} \mathrm{cm}^{-1}$ (biases $>50 \mathrm{~V}$ ), there appears to be an additional threshold for more complete EL2 ${ }^{+}$neutralization adjacent to the contact. The present study clearly demonstrates the often overlooked necessity of catering for built-in electric fields in positron diffusivity studies of III-V semiconductors where surface midgap Fermi-level pinning is common. (C) 1997 American Institute of Physics. [S0021-8979(97)02920-4]
\end{abstract}

\section{INTRODUCTION}

Recently metal/semi-insulating (SI)-GaAs constants have attracted both scientific and technological interest. ${ }^{1-5}$ Some of the SI-GaAs Schottky contact devices, such as radiation detectors, require operation under large reverse bias conditions and thus the distribution of the electric field at such contacts has become an important area of study. ${ }^{3-5}$ The positron, being a positive carrier of electric charge that possesses a simpler band structure and a relatively larger effective mass than its hole counterpart, ${ }^{6}$ can be usefully employed as an effective electric field probe within a semiconductor since the drift motion of the particle can be detected in a number of ways. ${ }^{7,8}$ In the present work, we present data taken with a low energy positron beam that largely confirms the emerging picture of an anomalous saturating electric field effect at the metal/SI-GaAs interface that appears to have its origin in an electric field enhancement of the EL2 electron capture cross section. ${ }^{4,5}$

The positron drift velocity $v_{+}$in an electric field $\xi$, as with its electron and hole counterparts, is characterized by the low field mobility $\left(\mu_{+}=v_{+} / \xi\right)$, the value of which may either be obtained from bulk samples with applied electric fields using positron lifetime spectroscopy ${ }^{9}$ or the Doppler shift technique, ${ }^{10}$ or be inferred from positron low energy positron beam derived diffusion length data by way of the Einstein relation. ${ }^{11}$ The limited available positron mobility data for semiconductors, however, shows a larger than expected scatter. In the case of diffusion length derived values, this has been attributed to the presence of "built-in" electric fields, which for the most part are uncertain and often ignored. ${ }^{12}$ Likewise, for bulk mobility studies, the electric

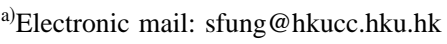

field is not always constant and this, if not correctly accounted for, leads to a systematic error., ${ }^{7,9,13}$ This highlights a current problem in dealing with positron electric field motion, namely that one either makes some assumption about the electric field structure within a sample and infers from the data the positron mobility, or conversely if the positron mobility is established, the particle may be used to obtain information regarding the electric field. If neither the mobility nor the electric field is well known, as is often the case, then great care must be taken in interpreting experimental data.

Ideally speaking, the positron mobility in a semiconductor, should be obtained independently of the diffusivity in a positron beam experiment by the application of an electric field to the sample under investigation. Such an approach is not only recommended because it is more direct but also because the variation of applied bias facilitates a larger data set for testing the drift-diffusion assumption and furthermore uncertainties due to built-in fields become less important. Mäkinen et al. successfully employed this approach to measure the positron mobility in $\mathrm{Si}$, where they applied the electric field to the sample by way of a $100 \AA$ Au epilayer, and analyzed the positron drift in the abrupt depletion model approximation. ${ }^{12}$ A $300 \mathrm{~K}$ mobility value for Si was obtained in good agreement with that obtained from other techniques. More recently the room temperature positron diffusion coefficient and mobility have been obtained for SI-GaAs using a similar methodology. ${ }^{14}$ Electric fields were applied by way of a $1000 \AA$ Au epilayer and were modeled with single valued mean electric field approximation over the depletion region. Unlike the case of $\mathrm{Si}$, however, this preliminary study revealed some anomalies. Not only was the $300 \mathrm{~K}$ mobility, $70 \pm 10 \mathrm{~cm}^{2} \mathrm{~V}^{-1} \mathrm{~s}^{-1}$, found to be significantly higher than the $40 \pm 10 \mathrm{~cm}^{2} \mathrm{~V}^{-1} \mathrm{~s}^{-1}$ value expected from bulk electric 
field measurements and theoretical calculation, ${ }^{7}$ but the positron beam data were found to saturate above about $25 \mathrm{~V}$, a fact that could not easily find explanation on the mean depletion region electric field model in which the electric field continues to increase as the square root of the applied bias.

In this work, we present further positron beam results for the $\mathrm{Au} / \mathrm{SI}-\mathrm{GaAs}$ and $\mathrm{Ni} / \mathrm{SI}-\mathrm{GaAs}$ systems under a wider range of reverse bias $(0-100 \mathrm{~V})$. The approach to data analysis is different in an important aspect from that of Refs. 12 and 14 in that we do not assume the abrupt depletion model, thereby imposing a square root of bias dependence on the mean electric field. Instead we find it preferable to reverse the procedure by attempting to extract from the data the mean electric field seen by the positron at the interface. In adopting this approach, it is possible to clearly see that the trend of the interfacial electric field is indeed that of tending towards an anomalous saturation above $25 \mathrm{~V}$. A lack of knowledge regarding the built-in field at zero bias, however, leads to some uncertainty in the magnitude of the saturation electric field. In appealing to saturation electric field data taken by direct probing ${ }^{4}$ and inferred from alpha particle implantation experiments, ${ }^{5}$ we are able to infer a positron mobility value of around $32 \pm 3 \mathrm{~cm}^{2} \mathrm{~V}^{-1} \mathrm{~s}^{-1}$ for SI-GaAs and the presence of an interfacial electric field of 2.0 $\pm 0.5 \mathrm{kV} \mathrm{cm}^{-1}$ at zero bias.

This article is structured as follows. In Sec. II, we find it helpful to review the present knowledge of the anomalous (nondepletion approximation) electric field structure at the metal-SI-GaAs interface. In Sec. III, experimental details of the positron beam experiment are given. The results are then presented and discussed in Sec. IV with conclusions being drawn in Sec. V.

\section{THE ELECTRIC FIELD DISTRIBUTION}

In undoped SI-GaAs, the concentration $N_{A}$ of residual acceptors (predominantly $\mathrm{C}$ impurities) are present in much higher concentration than residual shallow donors. The EL2 native defect deep donor, however, being present in still greater concentration causes the acceptors to become essentially fully ionized by pinning the Fermi energy of the GaAs around midgap. ${ }^{15}$ Because the Fermi energy at the metal/SIGaAs interface is pinned lower in the band gap than in the bulk, a surface electrostatic field forms. ${ }^{16}$ Under these conditions, the distribution of the electrostatic potential $\varphi(x)$ and the electric field $\xi(x)[=-d \varphi / d x]$ on the semiconductor side of the metal/SI-GaAs contact, is determined by Poisson's equation ${ }^{1,17}$

$$
\frac{d^{2} \varphi}{d x^{2}}=-\frac{d \xi}{d x}=-\frac{e}{\epsilon_{r} \epsilon_{0}}\left[N_{D D}^{+}(x)-N_{A}^{-}(x)-n(x)+p(x)\right]
$$

Here $\epsilon_{r}$ is the relative permittivity, and assuming thermal equilibrium between the depletion region and the bulk

$$
\begin{aligned}
& \begin{aligned}
& N_{A}^{-}(x)= N_{A}\left\{1+g_{A} \exp \left[\left(E_{A}-E_{F}-e \varphi(x)\right) / k T\right]\right\}^{-1}, \\
& \begin{aligned}
N_{D D}^{+}(x)= & N_{D D}\left\{1+g_{D D} \exp \left[\left(E_{F}-E_{D D}\right.\right.\right. \\
& +e \varphi(x)) / k T]\}^{-1},
\end{aligned} \\
& n(x)=n_{0} \exp (e \varphi(x) / k T), \text { and } \\
& p(x)=p_{0} \exp (-e \varphi(x) / k T)
\end{aligned}
\end{aligned}
$$

are the ionized acceptor, the ionized deep donor (EL2), the electron and the hole concentrations, respectively, at a depth $x$ into the substrate, $g_{A}$ and $g_{D D}$ are the degeneracy factors for the acceptor and deep donor, respectively, $E_{A}$ and $E_{D D}$ are the energy levels of the acceptor and deep donor, respectively, and $n_{0}$ and $p_{0}$ are the bulk equilibrium values of the electron and hole densities, respectively. Integration of Eqs. (1) and (2) may be carried out analytically. ${ }^{18}$ In the semiinsulator, where $n$ and $p$ are insignificant compared to the ionized donor and acceptor concentrations, this solution approximates to

$$
\begin{aligned}
\xi= & {\left[\frac{2 k T N_{D D}}{\epsilon_{r} \epsilon_{0}}\right]^{1 / 2}\left\{\ln \left[\frac{\exp (-e \varphi / k T)+f_{D D}}{1+f_{D D}}\right]\right.} \\
& \left.-\frac{N_{A}}{N_{D}} \frac{e \varphi}{k T}\right\}^{1 / 2},
\end{aligned}
$$

where $f_{D D}=g_{D D} \exp \left[\left(E_{F}-E_{D D}\right) / k T\right]$. Numerical integration of (3) with respect to $x$ can then give $\varphi(x)$, which when differentiated with respect to $x$ gives the required form of $\xi(x) .{ }^{1,19}$ This form of solution is tedious, not very tractable, and furthermore is based on the false assumption that the quasi-Fermi energies for electrons and holes remain constant and equal to the bulk Fermi energy position right up to the metal junction. ${ }^{20}$ Moreover, it is implicit in Eq. (2) that the electron capture rate for the ionized EL2 defect is independent of electric field, and, as reviewed below, there is mounting evidence for this not being the case. ${ }^{5,21}$ The form of Eq. (3) is nevertheless useful in modeling the Debye tail (low field region) at the metal-SI-GaAs junction in the present study.

The model that has often been applied in the past to biased SI-GaAs junctions ${ }^{7,13,14,22}$ is the solving of Eq. (1) under the depletion approximation. ${ }^{20}$ The justification behind the use of this simple model is that, providing the electron capture rate of the deep donor is electric field independent, the deep donor will become completely ionized $\left(N_{D D}^{+}\right.$ $\approx N_{D D}$ ) near the reverse biased Schottky junction thus forming a region of positive space charge defined by the net positive charge concentration $\left(N_{D D}-N_{A}\right)$. The transition to this fully ionized region is viewed as abrupt, whereas in reality the Debye tail can extend $\sim 0.5 \mu \mathrm{m}$ at $300 \mathrm{~K} .{ }^{19}$ Under these assumptions, Eq. (1) gives for the electric field at the contact, ${ }^{13,14,20}$

$$
\xi(x)=\frac{e\left(N_{D D}-N_{A}\right)}{\epsilon_{r} \epsilon_{0}}(w-x),
$$

where $w$, the bias-dependent width of the depletion region, is given by 


$$
w=\left[\frac{2 \epsilon_{r} \epsilon_{0}\left(\phi_{\mathrm{bi}}+V-I R_{b}\right)}{q\left(N_{D D}-N_{A}\right)}\right]^{1 / 2}
$$

$\phi_{\mathrm{bi}}\left[=\phi_{b}-\left(E_{C}-E_{F}\right)\right]$ being the built-in contact potential $\phi_{b}$ the Schottky barrier height, $E_{C}-E_{F}$ the bulk Fermi level measured from the bottom of the conduction band, $V$ the applied bias, $R_{b}$ the bulk resistance, and $I$ the current flowing through the sample.

According to Eq. (5), a bias of several thousand volts should be required to produce a depletion region $100 \mu \mathrm{m}$ wide. However, the collection of charge in alpha particle irradiation experiments clearly reveals that this is not the case and that the formation of such a width requires a bias of only $\sim 100 \mathrm{~V} .^{5}$ This surprising result is backed by independent direct measurements of the electric potential inside the biased metal/SI-GaAs contact. ${ }^{4}$ The explanation offered by McGregor et al. ${ }^{5}$ of this anomalous electric field distribution is that as the electric field approaches a critical value of $\sim 10 \mathrm{kV} \mathrm{cm}^{-1}$ a strong enhancement occurs in the electron capture cross section of the ionized native defect $\mathrm{EL} 2^{+}$, which essentially prevents any higher fields from forming. Independent evidence for an enhancement in capture cross section has been documented by Prinz and Rechkunov ${ }^{21}$ and the magnitude of the enhancement $\left(10^{-15} \mathrm{~cm}^{-2}\right.$ at low field to $10^{-13} \mathrm{~cm}^{-2}$ at fields above $10 \mathrm{kV} \mathrm{cm}^{-1}$ ) is sufficient to explain the anomaly. ${ }^{5}$ The increased capture by the EL2 ${ }^{+}$ sites towards electrons injected into the high field region over the Schottky-like barrier causes the ionized EL2 ${ }^{+}$concentration to decrease to a value close to that of the ionized shallow acceptors. Thus a condition sets in, in which the difference between the ionized deep donor and acceptor concentrations is small, causing a "quasineutral region" of low net space charge to form between the metal and a buried residual region of $\mathrm{EL}^{+}$space charge. McGregor et al. model this phenomenon by suggesting a relationship between the ionized EL2 concentration and the electric field of the form ${ }^{5}$

$$
\begin{aligned}
N_{D D}^{+}-N_{A}^{-}= & N_{D D}\left\{1+2 \exp \left[\left(E_{F}-E_{D D}\right.\right.\right. \\
& +q \varphi(x)) / k T]\}^{-1}-\left(N_{D D}-N_{A}\right) \\
& \times\left\{1+\left(\xi_{c} / \xi\right)^{\alpha}\right\}^{-1}-N_{A},
\end{aligned}
$$

where $\xi_{c}$ is a critical electric field for the onset of capture cross-section enhancement and the value of $\alpha$ characterizes how rapidly the trap filling occurs with the increasing electric field. The first term is the same as the $N_{D D}^{+}$expression in (2) assuming $g_{D D}=2$ and just represents the expected ionization for a field-independent capture cross section. The second term is added in an ad-hoc manner so as to simulate a rapid onset of deep donor neutralization when the field lies above $\xi_{c}$.

The form of Eq. (6) has two problems. The first is that the functional form, being both dependent on the electrostatic potential and the electric field, presents difficulty in numerical integration. The second is that the form can often go negative representing a greater concentration of ionized acceptors than ionized deep donors, and while this may indeed occur, there is little evidence that it does and our present understanding of the high field neutralization mecha-
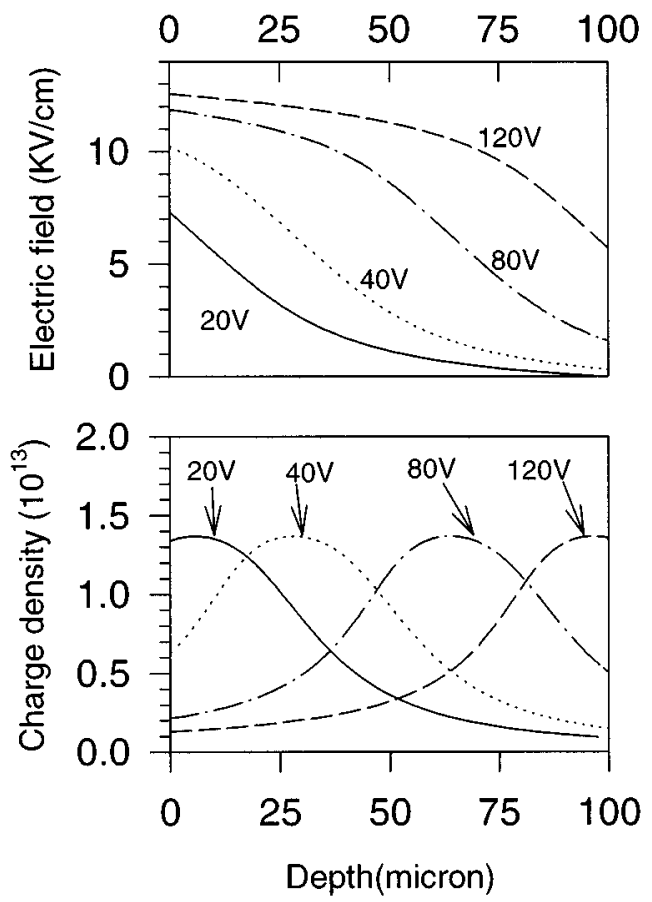

FIG. 1. Theoretical electric field and charge density distributions for the different applied bias voltages shown as based on the field enhanced EL2 ${ }^{+}$ capture cross-section model of McGregor et al. (Ref. 5), where $\alpha=10, \xi_{c}$ $=10 \mathrm{kV} \mathrm{cm}^{-1}$, and $\left(N_{D D}-N_{A}\right)_{\text {effective }}=1.4 \times 10^{13} \mathrm{~cm}^{-3}$.

nism of the space charge region is too limited to justify the modeling of any such inversion. The procedure we adopt in addressing these problems is to take a closely related and simpler form for the net charge density as follows

$$
\begin{aligned}
N_{D D}^{+}-N_{A}^{-}= & \left(N_{D D}-N_{A}\right)\left\{1+2 \exp \left[\left(E_{F}-E_{D D}\right.\right.\right. \\
& +q \varphi(\xi)) / k T]\}^{-1}\left\{1+\left(\xi / \xi_{C}\right)^{\alpha}\right\}^{-1},
\end{aligned}
$$

where $\varphi(\xi)$ is obtained by inversion of Eq. (3). In adopting this form, we have implied that within the low field freecarrier tail region there is no appreciable increase in electron capture, and therefore the relationship between $\varphi$ and $\xi$ will be very close to that given by Eq. (3). Equation (7) still presents the same fast tendency towards neutralization at electric fields higher than $\xi_{C}$ with the advantage of preventing the net charge density from becoming negative. The electric field distribution (positive fields being taken in the negative $x$ direction) can now be obtained by integration of Eq. (1):

$$
\frac{\epsilon_{r} \epsilon_{0}}{e} \int_{\xi(x)}^{\xi_{\max }} \frac{d \xi}{\left[N_{D D}^{+}-N_{A}^{-}\right]}=x,
$$

where, $\xi_{\max }$, the maximum electric field at $x=0$, is given by

$$
\begin{aligned}
\frac{\epsilon_{r} \epsilon_{0}}{e} \int_{0}^{\xi_{\max }} \frac{\xi d \xi}{\left[N_{D D}^{+}-N_{A}^{-}\right]} & =-\int_{0}^{-\left(\phi_{\mathrm{bi}}+V-I R_{b}\right)} d \varphi \\
& =V+\phi_{\mathrm{bi}}-I R_{b} .
\end{aligned}
$$

To demonstrate the reasonable validity of this form of solution, we set $\xi_{c}=10^{4} \mathrm{~V} / \mathrm{cm}, \alpha=10$ and $N_{D D}-N_{A}=1.4$ $\times 10^{13} \mathrm{~cm}^{-3}$ and show in Fig. (1) the calculated charge den- 
sity $N_{D D}^{+}-N_{A}^{-}$and electric field profiles as obtained for different biases. At the higher biases, it is noted that the electric field remains reasonably constant up to a certain depth, the value of which varies in approximate proportion to the applied bias. Beyond this point, the electric field profile exhibits a steeper decent due to the presence of the buried EL2 ${ }^{+}$ region. This form of electric field profile is very similar to that observed experimentally by Berwick et al. ${ }^{4}$ It is also similar to the essentially identical model of McGregor et al. ${ }^{5}$ in that the depletion region expands at the same rate with applied bias. The fall off in charge at the depletion zone edge, of our simulation is, however, less steep than that of Ref. 5, because we have artificially set $N_{D D}-N_{A}$ three orders of magnitude lower than the anticipated value of $\sim 1.4$ $\times 10^{16} \mathrm{~cm}^{-3}$, in order to bring some approximate agreement with the data of Ref. 4, which clearly show a much broader width $(30-100 \mu \mathrm{m})$ to the depletion zone edge " $90 \%-10 \%$ fall-off"' region. In the discussion below, we show that our present positron beam data strongly confirm this low concentration of net positive charge within the buried EL2 ${ }^{+}$layer. One can conclude from these observations that not only is there a very rapid onset of essentially complete EL2 ${ }^{+}$ neutralization at high field $>\sim 10 \mathrm{kV} \mathrm{cm}^{-1}$, but at much lower fields there is also a significantly increased capture rate.

\section{EXPERIMENT}

The substrate used in this study was undoped liquidencapsulated Czochralski (LEC) grown SI-GaAs(100). The wafer was purchased from ICI Wafer Technology Ltd and had a room temperature resistivity of $8 \times 10^{7} \Omega \mathrm{cm}$, a thickness of $500 \mu \mathrm{m}$, and an EL2 concentration of 1.5 $\times 10^{16} \mathrm{~cm}^{-3}$. A $10 \times 10 \mathrm{~mm}^{2}$ square section of the material was cut and then degreased in acetone and ethanol before being etched in standard $\mathrm{NH}_{4} \mathrm{OH} . \mathrm{H}_{2} \mathrm{O}_{2}: \mathrm{H}_{2} \mathrm{O}(3: 1: 9)$ and $\mathrm{H}_{2} \mathrm{SO}_{4}: \mathrm{H}_{2} \mathrm{O}_{2}: \mathrm{H}_{2} \mathrm{O}(8: 1: 1)$ solution for 1 min. A $1000 \AA \mathrm{Au}$ film was then evaporated onto each side of the substrate in turn using electron beam evaporation in a vacuum of $10^{-6}$ mbar. A circular aperture was used to confine the film to circular spots of size $8 \mathrm{~mm}$ diam. Electrical connection to the circular films was by way of thin Au wires attached by a small amount of silver paint. As in previous studies, ${ }^{13,14}$ the $\mathrm{Au} / \mathrm{SI}$-GaAs contacts, were verified to be of a Schottky-like (rectifying) nature, by taking (I-V) characteristics.

The positron beam experiment consisted of implanting positrons of controlled energy into a reverse biased contact where the internal electric field direction was opposite to the direction of positron injection, and such as to cause drift back to the Au/SI-GaAs interface. The positron annihilation spectroscopy measurements were carried out using the magnetically guided positron beam at The University of Hong Kong which has been described in Ref. 22. The intensity of the slow positron beam was about $1 \times 10^{5} \mathrm{e}^{+} / \mathrm{s}$, and its diameter was $6 \mathrm{~mm}$. The incident-beam energy was varied from 0.15 to $25 \mathrm{keV}$ in steps of $250 \mathrm{eV}$. The $511 \mathrm{keV}$ annihilation $\gamma$ spectra were detected and accumulated by a highpurity Ge detector of resolution $1.4 \mathrm{keV}$ at $514 \mathrm{keV}$ and a digitally stabilized multichannel analyzer system. A total of

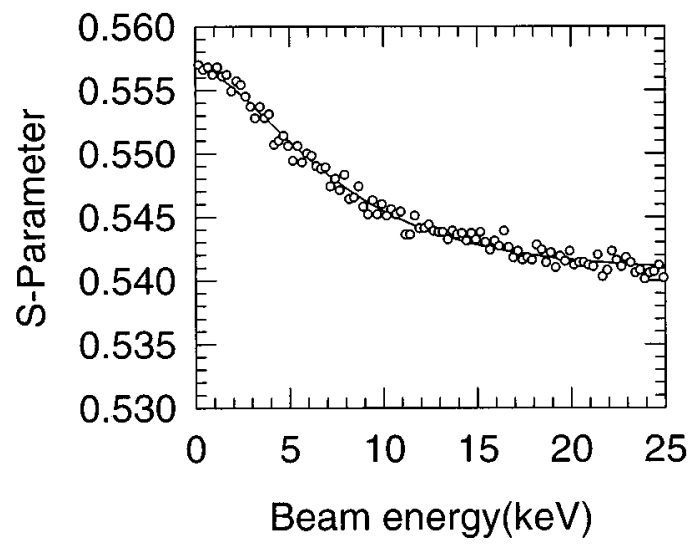

FIG. 2. The $S(E)$ spectra for the SI-GaAs substrate. The fitted diffusion length is $2050(50) \AA$.

$1 \times 10^{6}$ counts were collected under the annihilation photopeak for each positron energy. The photopeak line shape was characterized by way of the $S$ parameter which was calculated in the normal way by dividing the central region of the $511 \mathrm{keV}$ peak by the total peak counts. ${ }^{11} S$ parameter versus implantation energy data were taken at every $10 \mathrm{~V}$ of reverse bias up to a maximum bias of $100 \mathrm{~V}$ for both the Ni/SI-GaAs and Au/SI-GaAs samples. The current through the sample was monitored at each bias.

\section{RESULTS AND DISCUSSION}

In Fig. 2, we plot the line-shape parameter " $S$ " as a function of the incident positron beam energy before evaporation of the Au film onto the substrate's surface. The general form of this curve is as seen by others ${ }^{23,24}$ and has a simple explanation in terms of the competing sites of annihilation, namely those from the surface and the bulk states which have the different characteristic $S$ values $S_{S}$ and $S_{B}$, respectively. This fact is expressed by writing ${ }^{11}$

$$
S(\mathrm{E})=f(\mathrm{E}) S_{S}+[1-f(\mathrm{E})] S_{B},
$$

where $f(\mathrm{E})$, the fraction of positrons implanted at energy $\mathrm{E}$ that drift diffuse back to the surface, is proportional to Laplace transform of the implantation profile $P(\mathrm{E}, x):{ }^{24,11}$

$$
f(\mathrm{E}) \propto \int_{0}^{\infty} P(\mathrm{E}, x) e^{-x / L_{B}^{\mathrm{eff}}} d x .
$$

The fall in $f(\mathrm{E})$ is thus characterized by the material's effective positron diffusion length $L_{B}^{\text {eff }}$. At low implantation energies, the $S$-parameter is high because it is more characteristic of the surface positron state. As the energy increases further, the $S$ parameter drops to $S_{B}$ as $f(\mathrm{E})$ becomes progressively smaller. The fact that $S_{S} \gg S_{B}$ is as expected; first, because positrons at the surface experience less compression into interstitial regions thus experiencing less high momentum core annihilations, and second because para- $P s$ can form at the GaAs surface. ${ }^{25}$

Shown in Figs. 3 and 4 are the $S(\mathrm{E})$ spectra taken for the $\mathrm{Au} / \mathrm{SI}-\mathrm{GaAs}$ and Ni/SI-GaAs systems, respectively, under different reverse biases. The shape of the curves differs notably from that of the substrate as a result of the metalization 


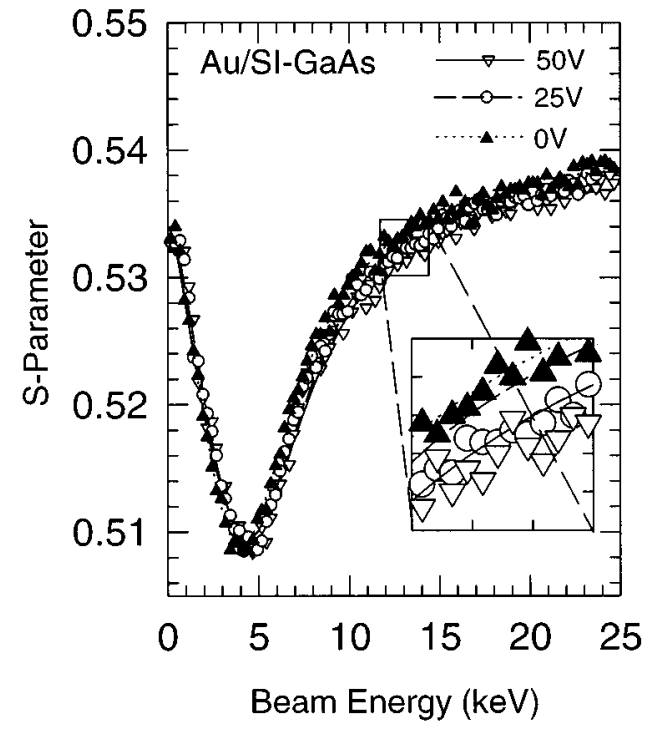

FIG. 3. The $S(E)$ spectra for the Au/SI-GaAs system for reverse biases of 0 , 25 , and $50 \mathrm{~V}$. The thickness of the Au overlayer is $1000 \AA$. The curves shown are as modeled using VEPFIT.

and changes only a little under bias. At beam implantation energies of less than $1 \mathrm{keV}$, the $S$ value is comparatively high and characterizes positrons annihilating on the metal surface. As the energy increases, however, more positrons annihilate in the metal overlayer, for which the characteristic $S$ value is low and $S$ falls. For beam energies larger than $\sim 5 \mathrm{keV}$, the $S$ parameter begins to increase from its valley value, as a result of implanted positrons penetrating through the metal overlayer into the interface region and the bulk. The $S$ parameter then rises towards a saturation value as positrons predominantly annihilate in the GaAs bulk with $S$ parameter value $S_{B}$. Ling et al. have found it necessary to use a three layer model in the fitting $S(\mathrm{E})$ data for metal/SIGaAs systems. ${ }^{26}$ These consist of (i) the metal overlayer $\left(0<x<B_{1}, S=S_{0}\right)$, (ii) an interfacial layer $\left(B_{1}<x<B_{2}, S\right.$

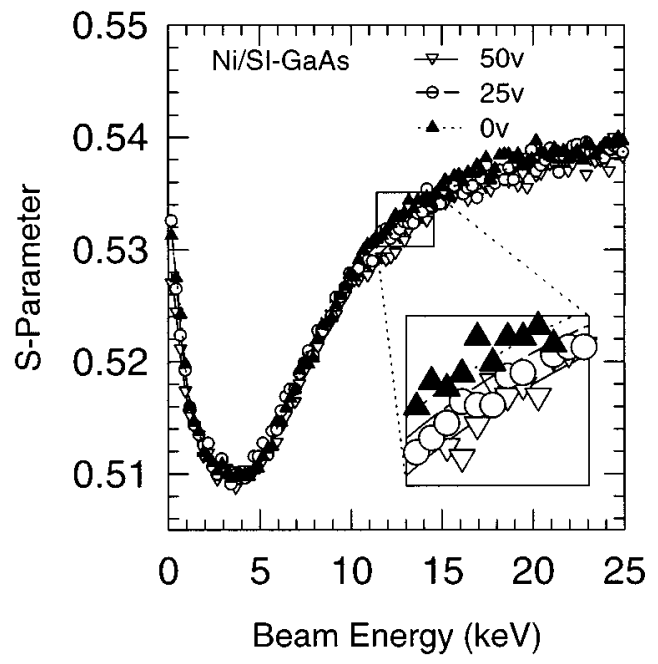

FIG. 4. The $S(E)$ spectra for the Ni/SI-GaAs system for reverse biases of 0 , 25 , and $50 \mathrm{~V}$. The thickness of the Ni overlayer being $1000 \AA$ A. The curves shown are as modeled using VEPFIT.
TABLE I. The values of the parameters used in the VEPFIT analysis.

\begin{tabular}{lcccc}
\hline \hline \multicolumn{1}{c}{ Materials } & & $\mathrm{GaAs}$ & $\mathrm{Au}$ & $\mathrm{Ni}$ \\
\hline Density & $\rho$ & $5.32 \mathrm{~g} \mathrm{~cm}^{-3}$ & $19.3 \mathrm{~g} \mathrm{~cm}^{-3}$ & $8.9 \mathrm{~g} / \mathrm{cm}^{-3}$ \\
Implantation & $\alpha$ & $450 \AA \mathrm{g} \mathrm{cm}^{-3 \mathrm{a}}$ & $831 \AA \mathrm{g} \mathrm{cm}^{-3 \mathrm{~b}}$ & $450 \AA \mathrm{g} \mathrm{cm}^{-3 \mathrm{a}}$ \\
parameters & $m$ & $2.0^{\mathrm{a}}$ & $2.0^{\mathrm{b}}$ & $2.0^{\mathrm{a}}$ \\
& $n$ & $1.6^{\mathrm{a}}$ & $1.42^{\mathrm{b}}$ & $1.6^{\mathrm{a}}$ \\
Thickness & $\Delta$ & $0.5 \mathrm{~mm}$ & $1000 \AA$ & $1000 \AA$ \\
\hline \hline
\end{tabular}

${ }^{a}=$ Ref. 28 .

$\mathrm{b}=$ Ref. 29 .

$\left.=S_{I}\right)$, and (iii) the semi-infinite bulk $\left(x>B_{2}, S=S_{B}\right) . B_{1}$ is the boundary between the overlayer and interface layer and $B_{2}$ is the boundary between the interface layer and the bulk region. The model can thus be expressed in the form

$$
\begin{aligned}
S(\mathrm{E})= & F_{S}(\mathrm{E}) S_{S}+F_{0}(\mathrm{E}) S_{0}+F_{I}(\mathrm{E}) S_{I}+\left(1-F_{S}(\mathrm{E})\right. \\
& \left.-F_{0}(\mathrm{E})-F_{I}(\mathrm{E})\right)\left\{f(\xi) S_{I}+[1-f(\xi)] S_{B}\right\},
\end{aligned}
$$

the $f(\xi)$ in this equation being the same as that expressed by Eq. (11) providing one may consider as abrupt the change from the overlayer to the bulk density at boundary $B_{2} \cdot{ }^{14}$ Under this approximation, we once again see the asymptotic approach to $S_{B}$ with increasing beam energy as being characterized by the effective positron diffusion length.

In the present work, no analytic forms are taken for fitting Eqs. (11) and (12) to the data. Instead we fit the $S(\mathrm{E})$ spectra using the VEPFIT software. ${ }^{27}$ The parameter values used for the standard Makovian form of implantation profile $P(\mathrm{E}, x)$ are given in Table I. For the data of Fig. 2, we find surface and bulk $S$ parameters of 0.558 and 0.538 , respectively, and a positron diffusion length $L_{B}^{\text {eff }}$ of $2050 \pm 50 \AA$. For the zero-biased data of Figs. 3 and 4, the fitted parameters are given in the Table I and the resulting fits to the data are shown by the curves in the figures. Throughout the fitting, the diffusion length in the extended interfacial region, $L_{I}$, was set equal to $0.01 \AA$ to simulate perfect absorption in this disordered region. ${ }^{26}$

We now address the question of the bias dependence. For the sake of clarity, only a few of the curves are shown on Figs. 3 and 4 (biases $=0,25$, and $50 \mathrm{~V}$ ). It is noted that the data show a small but definite lowering of the $S$ value in the $5-25 \mathrm{keV}$ range with increasing applied bias. This occurs as a result of a greater fraction $[f(\xi)$ increasing] of positrons being drifted into the interfacial region into sites that are probably open volumes (microvoids) at the interface. ${ }^{13}$ Since the extended interfacial region's $S$-parameter value is significantly lower than that of the bulk ${ }^{26,30}$ the net value of $S$, as expressed by Eq. (12), decreases. Moreover the effect is not linear with bias, with the difference between the 0 and $25 \mathrm{~V}$ data being noticeably larger than that between 25 and $50 \mathrm{~V}$ and although not shown in the figures, it is found that there is no visual difference between the $S(\mathrm{E})$ spectra above $50 \mathrm{~V}$ bias. It thus appears that the drifted positron fraction $f(\xi)$ is saturating at biases of around $50 \mathrm{~V}$. Two possible explanations may be forwarded. The first is that the depletion approximation is holding with full EL2 ionization giving rise to maximal electric fields $\left(2 N_{D D} e V / \epsilon_{r} \epsilon_{0}\right)^{1 / 2}$ $\sim 400 \mathrm{kV} \mathrm{cm}^{-1}$ and that such large fields are producing 
TABLE II. VEPFIT fitting results without bias.

\begin{tabular}{lcccccccc}
\hline \hline Sample & $S_{s}$ & $S_{0}$ & $S_{I}$ & $S_{B}$ & $\begin{array}{c}B_{1} \\
(\AA)\end{array}$ & $\begin{array}{c}B_{2} \\
(\AA)\end{array}$ & $\begin{array}{c}L_{0} \\
(\AA)\end{array}$ & $\begin{array}{c}L_{B} \\
(\AA)\end{array}$ \\
\hline Au/SI-GaAs & $0.5330(3)$ & $0.5082(3)$ & $0.5293(2)$ & $0.5396(1)$ & $970(50)$ & $1100(50)$ & $60(10)$ & $1950(50)$ \\
Ni/SI-GaAs & $0.5355(3)$ & $0.5099(1)$ & $0.5314(2)$ & $0.5396(4)$ & $950(50)$ & $1020(50)$ & $80(15)$ & $2250(70)$ \\
\hline \hline
\end{tabular}

positron drift velocities close to the expected optical phonon limit at around $\sim 10^{7} \mathrm{~cm} \mathrm{~s}^{-1}$. Such limiting velocities could possibly be achieved if the positron mobility exceeded $100 \mathrm{~cm}^{2} \mathrm{~V}^{-1} \mathrm{~s}^{-1}$. This explanation seems unlikely on a number of grounds. A saturation velocity of $\sim 10^{7} \mathrm{cms}^{-1}$ would be difficult to achieve even at such high fields with a more typical mobility value of $\sim 40 \mathrm{~cm}^{2} \mathrm{~V}^{-1} \mathrm{~s}^{-1}$ since application of the Shockley expression ${ }^{17,13}$ would indicate a more modest acoustic phonon limited drift velocity $\sim 3 \times 10^{6} \mathrm{~cm} \mathrm{~s}^{-1}$. Moreover the value of the electric field is close to the breakdown field in $\mathrm{GaAs}^{17}$ and were such fields really occurring at $50 \mathrm{~V}$ bias it would be difficult to understand how the bias across the sample could increase to $200 \mathrm{~V}$ or more, as is indeed possible in these samples. Finally the depletion width would be $\sim 2 \mu \mathrm{m}$ and with a drift velocity $\sim 10^{7} \mathrm{~cm} \mathrm{~s}^{-1}$ the positron transit time across the region would be only 20 ps. This being short compared to the 230 ps lifetime of positrons in bulk GaAs, ${ }^{31}$ would indicate effective diffusion lengths in excess of $2 \mu \mathrm{m}$, which are not observed. We are thus left with the conclusion that it is the saturation of the electric field that leads to the observed invariance of positron drift above $50 \mathrm{~V}$ bias. This second, and preferred interpretation, is in agreement with the behavior of the interfacial electric field observed in other recent experiments. ${ }^{4,5}$

In order to quantify the variation of the interfacial electric field with increasing applied bias, the experimental spectra taken with different biases were also analyzed using VEPFIT. The fits were constrained with the values of $S_{s}, S_{0}$, $S_{I}, L_{I}, B_{1}$, and $B_{2}$, being fixed at their zero bias values as given in Table II with only the positron diffusion lengths in the overlayer $L_{0}$ and bulk $L_{B}^{\text {eff }}$ being allowed to vary. Assuming the presence of some electric field $\xi$, that may be considered constant over the implantation region of the positron, the measured diffusion length $L_{B}^{\text {eff }}$ is the "effective diffusion length" which is related to the true (flat band) diffusion length $L_{B}$ according to the equation: ${ }^{32}$

$$
L_{B}^{\mathrm{eff}}=\frac{1}{-\frac{e \xi}{2 k T}+\left[\left(\frac{e \xi}{2 k T}\right)^{2}+\frac{1}{L_{B}^{2}}\right]^{1 / 2} .}
$$

Rearrangement of Eq. (13) gives the mean interfacial electric field as being

$$
\xi=\frac{k T}{e L_{B}^{\mathrm{eff}}}\left[\left(\frac{L_{B}^{\mathrm{eff}}}{L_{B}}\right)^{2}-1\right]
$$

In Fig. 5, we show this positron sampled interfacial electric field $\xi$ as a function of the applied bias calculated assuming different values for $L_{B}$ of 1250,1450 , and $2050 \AA$, respectively, the latter value being that observed in the fit to the substrate data of Fig. 2. Although the value chosen for
$L_{B}$ has a strong influence on the magnitude of the electric field, the saturation effect with the applied reverse bias is clearly evidenced in all these derived data. In the low bias region, the electric field increases with the increasing bias while for biases over $50 \mathrm{~V}$, the change of the electric field is considerably smaller. No significant difference is seen between the data derived from the $\mathrm{Au}$ and $\mathrm{Ni}$ metalizations, confirming that we are indeed observing the bulk transport property of the positron.

Among the derived data sets, the lower $(\mathrm{Au}, \mathrm{Ni})$ pair for $L_{B}=2050 \AA$ are those that one might initially look to in giving the desired interfacial $\xi(0)$ dependence on applied bias, by making the assumption that there is no intrinsic elec-

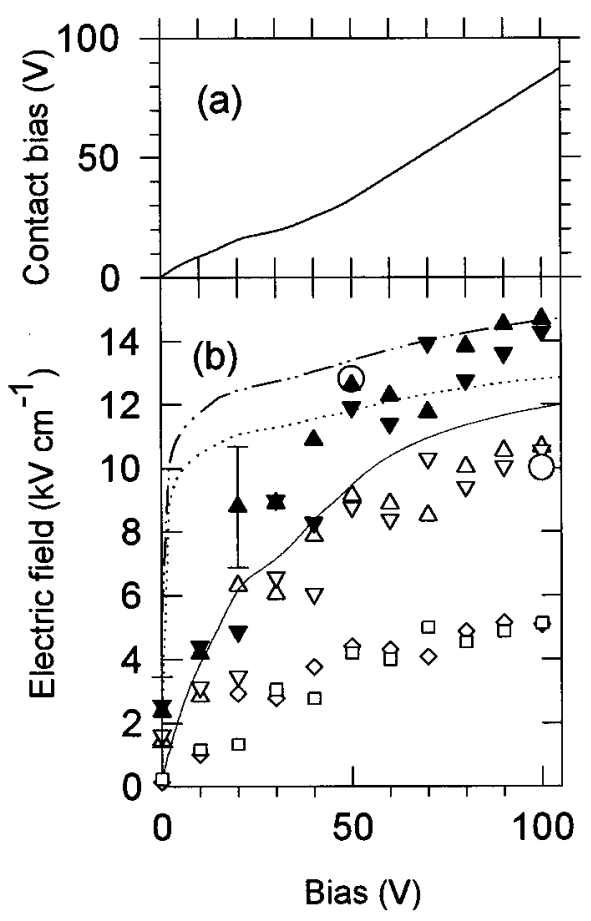

FIG. 5. The positron sensed electric field at the Au/SI-GaAs and Ni/SIGaAs. (a) The expected variation of the contact bias $\left(V-I R_{b}\right)$ as a function of applied bias. (b) The results of the positron sensed electric field for three values of the flat-band diffusion length $L_{B}=2050 \AA,(\diamond=\mathrm{Au}$, $\square=\mathrm{Ni}) 1450 \AA(\triangle=\mathrm{Au}, \nabla=\mathrm{Ni}), 1250 \AA(\boldsymbol{\Delta}=\mathrm{Au}, \nabla=\mathrm{Ni})$. The data represented by the large open circles are taken from the experimental data of Berwick et al. (Ref. 4). The curves through the data are theoretically derived based on the model of McGregor et al. (Ref. 5) and the electric field dependent net charge density as expressed in Eq. (7). The best fit (solid line) is for $\left(N_{D D}-N_{A}\right)_{\text {effective }}=1.4 \times 10^{13} \mathrm{~cm}^{-3}, \alpha=10$, and $\xi_{C}=10 \mathrm{kV} \mathrm{cm}^{-1}$. The dotted line, which gives the correct zero-bias electric field, is for $\left(N_{D D}\right.$ $\left.-N_{A}\right)_{\text {effective }}=5.8 \times 10^{14} \mathrm{~cm}^{-3}, \alpha=10$, and $\xi_{C}=7 \mathrm{kV} \mathrm{cm}^{-1}$. The dot-dashed line is for $\left(N_{D D}-N_{A}\right)_{\text {effective }}=1.4 \times 10^{16} \mathrm{~cm}^{-3}, \quad \alpha=10$, and $\xi_{C}$ $=7 \mathrm{kV} \mathrm{cm}^{-1}$, corresponds to the case where there is no low-field enhanced capture. 
TABLE III. Values obtained by others on the positron mobility (diffusivity) in GaAs. Caption, BD=Beam method assuming just diffusion, $\mathrm{BDD}=$ Beam method assuming both drift and diffusion, DS $=$ Doppler shift technique, $\mathrm{LT}=$ Positron lifetime technique. The underlined value is the primary experimental value from which the other related parameters are deduced.

\begin{tabular}{|c|c|c|c|c|c|}
\hline $\begin{array}{l}L_{B}^{+} \\
(\AA)\end{array}$ & $\begin{array}{c}D_{+} \\
\left(\mathrm{cm}^{2} \mathrm{~s}^{-1}\right)\end{array}$ & $\begin{array}{c}\mu_{+} \\
\left(\mathrm{cm}^{2} \mathrm{~V}^{-1} \mathrm{~s}^{-1}\right)\end{array}$ & Sample & $\begin{array}{l}\text { Technique } \\
+ \text { comments }\end{array}$ & Researchers \\
\hline $1350(100)$ & $0.79(12)$ & $32.5(5.0)$ & Undoped SI & BDD & Present work \\
\hline$\overline{1400}$ & 0.85 & 32.9 & $n$ type & $\mathrm{BD}$ & $\begin{array}{l}\text { Saarinen et al. } \\
\text { Ref. } 34\end{array}$ \\
\hline $1447(200)$ & $0.91(26)$ & $35(10)$ & Undoped SI & DS & Au et al. Ref. 35 \\
\hline $1547(200)$ & $1.04(26)$ & $\overline{40}(10)$ & Undoped SI & DS + Theory $^{\mathrm{a}}$ & Au et al. Ref. 7 \\
\hline $1800(140)$ & $1.4(2)$ & $\overline{54}(8)$ & Undoped SI & $\mathrm{BD}$ & Evans et al. Ref. 24 \\
\hline$\overline{1890}(490)$ & $1.55(52)$ & $60(20)$ & Undoped SI & $\mathrm{LT}$ & Shan et al. Ref. 13 \\
\hline 1920(120) & $\underline{1.6(2)}$ & $\overline{62}(8)$ & $\begin{array}{l}\text { SI-LEC }+ \\
n \text { type } 2 \times 10^{14}\end{array}$ & BD+Theory & $\begin{array}{l}\text { Soininen et al. } \\
\text { Ref. } 33\end{array}$ \\
\hline $2035(145)$ & $1.8(3)$ & $\underline{70}(10)$ & Undoped SI & BDD & Shan et al. Ref. 14 \\
\hline$\underline{2050(50)}$ & $1.83(9)$ & $\overline{70} .6(34)$ & Undoped SI & $\begin{array}{l}\text { BD No } \xi \text {-field } \\
\text { correction }\end{array}$ & present work \\
\hline $2270(100)$ & $2.24(20)$ & $87(8)$ & Undoped SI & $\mathrm{BD}$ & Ling et al. Ref. 26 \\
\hline$\overline{2900}$ & 3.66 & 141 & $\mathrm{Be}^{+}$implanted & $\mathrm{BD}$ & Uedono et al. Ref. 23 \\
\hline$\overline{2900}(100)$ & $3.66(25)$ & 141(10) & $?$ & $\mathrm{BD}$ & Lee et al. Ref. 36 \\
\hline$\overline{7237(157)}$ & $23(1)$ & $\underline{880(40)}$ & Undoped SI & $\begin{array}{l}\text { LT Constant } \\
\xi \text {-field approx }\end{array}$ & Yu and Cao Ref. 37 \\
\hline
\end{tabular}

${ }^{a}$ Values of between 80 and $100 \mathrm{~cm}^{2} \mathrm{~V}^{-1} \mathrm{~s}^{-1}$ are obtained in Ref. 7, but as pointed out in that work this is only an apparent value due to the electric field structure in the samples.

tric field present in the substrate control experiment (Fig. 2). This indeed is the approach most often adopted by positron beam workers in the past and, as shown in Table III, does lead to derived mobility values in the range of $50-140 \mathrm{~cm}^{2} \mathrm{~V}^{-1} \mathrm{~s}^{-1}$. Such values are, on average, higher than theory or those derived from the more direct Doppler shift method (range of $30-60 \mathrm{~cm}^{2} \mathrm{~V}^{-1} \mathrm{~s}^{-1}$. ${ }^{7,33}$ There are two reasons that lead us to believe that the assumption of a zero electric field (flat bands) in a nonbiased sample is incorrect and such as to cause a systematic error. The first is that it is known that the presence of oxide causes surface Fermi level pinning within the range of $0.7-0.9 \mathrm{eV}$ below the conduction band in $n$ - and $p$-type GaAs. ${ }^{20}$ A similar surface pinning position would thus be expected for SI-GaAs, where the bulk Fermi energy lies 0.6 to $0.7 \mathrm{eV}$ below the conduction band. ${ }^{34}$ Thus a small upward band bending in the range of $0-0.3 \mathrm{eV}$ is expected at the oxided SI-GaAs surface which, as is shown below, could produce an intrinsic electric field capable of increasing the positron diffusion length to the observed effective value. The second argument is, however, more convincing; namely that if one takes a significantly shorter value of $L_{B}$, then it becomes immediately possible to attain data that are consistent with the kind of saturated electric fields $\sim 12 \mathrm{kV} \mathrm{cm}^{-1}$ seen by Berwick et al. ${ }^{5}$ and as inferred by McGregor et al. ${ }^{4}$ at the metal/SI-GaAs interface. This may be seen with reference to the upper two pairs of data sets in Fig. 5, which correspond to $L_{B}$ values of 1250 and $1450 \AA$. Standardizing in this way on the saturated electric field value indicated by Refs. 4 and 5 would thus suggest a flat-band $L_{B}$ value of $1350 \pm 100 \AA$, which through the relationship $L_{B}$ $=\sqrt{D_{+} \tau_{+}}$and the Einstein equation $\left(\mu_{+}=e D_{+} / k T\right)$ would equate with a mobility value of $32.5 \pm 3 \mathrm{~cm}^{2} \mathrm{~V}^{-1} \mathrm{~s}^{-1}$, a value which is in much better agreement with mobility values obtained from theory ${ }^{7,33}$ and using the Doppler shift technique. $^{7,35}$ In this context, it is perhaps noteworthy that the only other low value of $L_{B}(=1400 \AA)$ derived using the positron beam drift technique is that obtained on $n$-doped material, in which the intrinsic depletion depth may well be too small to cause any noticeable effect on positron diffusion. ${ }^{34}$

To complete the discussion, we fit the positron sampled interfacial electric field data to the field enhanced EL2 ${ }^{+}$capture cross-section model outlined in Sec. III. It expected that the mean effective positron diffusion length in the present experiment should, in the first approximation, be conditioned by the electric field at the metal/SI-GaAs interface; namely $\xi(0)$. In Fig. 5, we show the model fit for $\xi(0)$ as derived using Eqs. (7)-(9) with $\xi_{c}=10^{4} \mathrm{~V} / \mathrm{cm}, \alpha=10$ and $\left(N_{D D}\right.$ $\left.-N_{A}\right)_{\text {effective }}=1.4 \times 10^{13} \mathrm{~cm}^{-3}$. In this fit, we have taken the resistance drop across the bulk into account with a bulk resistivity $R_{b}$ of $10^{7} \Omega$ and a current as interpolated from experimental values. ${ }^{13}$ The built-in band bending has been taken as $0.16 \mathrm{eV}$ as has been observed experimentally for the $\mathrm{Ni} / \mathrm{SI}-\mathrm{GaAs}$ interface. ${ }^{16}$ The general trend of the model fit is good except at zero bias where the experimentally observed field is higher than predicted by the model. At all higher biases, however, the model lies in between the 1250 and $1450 \AA$ data sets, and is thus in accord with the direct experimental data of Ref. 4.

It is interesting to note that if we choose a $\left(N_{D D}\right.$ $\left.-N_{A}\right)_{\text {effective }}$ value close to the anticipated value $N_{D D}-N_{A}$ $=1.4 \times 10^{16} \mathrm{~cm}^{-3}$ a very poor fit to the data is found (see Fig. 5). Not only does the zero bias interfacial field greatly exceed the observed value, but the approach to the saturation field is far too quick. Conversely, it is only when we take a $\left(N_{D D}-N_{A}\right)_{\text {effective }}$ close to $10^{13} \mathrm{~cm}^{-3}$ that we can get the same rate of rise of $\xi(0)$ with increasing bias as found in our experiment. With this value being very similar to that in- 
ferred from the observed gradient to the electric field data of Ref. 4, we consider this as giving strong evidence that the depletion zone at the reverse biased metal/SI-GaAs interface is far from being fully ionized. This unusual phenomenon finds natural explanation if there is already some increased capture onto the $\mathrm{EL}_{2}{ }^{+}$site even at low electric fields (mean $\sim 1 \mathrm{kV} \mathrm{cm}^{-1}$ ). One very likely causation for this behavior, which has been suggested by Johnson, ${ }^{39}$ is that the Gunn effect, which has a threshold field of $3.2 \mathrm{kV} \mathrm{cm}^{-1}$ in $\mathrm{GaAs},{ }^{17}$ is beginning to take place. The EL2 ${ }^{+}$capture cross section is seen as being much greater for electrons that have been excited into the $L$ band through the action of the electric field. ${ }^{39}$

For a fully ionized, zero biased SI-GaAs system ${ }^{19}$ having a band bending of $\sim 0.2 \mathrm{eV}^{16}$ fields $\left(\sim 20 \mathrm{kV} \mathrm{cm}^{-1}\right)$, which are well in excess of the Gunn effect threshold field, are to be expected. Some space charge neutralization would thus be anticipated even at zero bias on the Johnson model. ${ }^{39}$ In this context, we note that our electric field measurement at zero bias $\left(2 \mathrm{kV} \pm 0.5 \mathrm{kV} \mathrm{cm}^{-1}\right)$ is noticeably less than that predicted on the full ionization picture of Ref. 19 which gives, as seen from Fig. 5, $\xi(0) \sim 5 \mathrm{kV} \mathrm{cm}^{-1}$. On the other hand, as also seen from Fig. 5, the value we observe for the zero bias interfacial field is larger than predicted with the $\left(N_{D D}\right.$ $\left.-N_{A}\right)_{\text {effective }}$ value of $1.4 \times 10^{13} \mathrm{~cm}^{-3}$. This indicates that some low-field neutralization effect has become operative even at zero bias, and, since a value of $\left(N_{D D}-N_{A}\right)_{\text {effective }}$ $=5.8 \times 10^{14} \mathrm{~cm}^{-3}$ is required to reproduce the observed electric field (see Fig. 5), the EL2 ionization is only at the $4 \%$ level. Since the model fit with $\left(N_{D D}-N_{A}\right)_{\text {effective }}=1.4$ $\times 10^{13} \mathrm{~cm}^{-3}$ becomes reasonable at $10 \mathrm{~V}$ it may be concluded that the low-field neutralization effect has saturated at this bias with a resulting drop in the EL2 ionization to the $0.1 \%$ level. These facts appear consistent at least with the low-field neutralization process being linked to the Gunn effect, in the manner suggested by Johnston. ${ }^{39}$

\section{CONCLUSIONS}

A variable energy positron beam has been used to study the bias dependence of the interfacial electric field at the metal/SI-GaAs interface. This has been made possible by the fact that at the metal/SI-GaAs contact under reverse bias, the positrons are injected in opposition to the direction of the electric field, which aids their drift-diffusion motion back to the extended interfacial region at which position they trap causing an observable change of $S$ parameter. Based on the experimental results, the saturation effect of the electric field at the metal/SI-GaAs interface, as seen recently by other workers using different techniques, has been confirmed. The experimental results clearly indicate that, at small reverse bias $(<50 \mathrm{~V})$, the interfacial electric field increases rapidly with applied bias, but that for reverse biases above $50 \mathrm{~V}$, the increase is considerably smaller. The positron data are consistent with a saturation field of $12 \pm 2 \mathrm{kV} \mathrm{cm}^{-1}$ and a positron mobility value of $32.5 \pm 3 \mathrm{~cm}^{2} \mathrm{~V}^{-1} \mathrm{~s}^{-1}$.

The zero bias residual interfacial electric field has been found to be $2 \pm 0.5 \mathrm{kV} \mathrm{cm}^{-1}$. It is believed that residual fields of this magnitude are to be generally found at oxidized SI-GaAs substrates and that this has caused a systematic er- rors in much of the past positron beam work on SI-GaAs. This indicates that more care should be taken in future positron beam studies to compensate for intrinsic electric fields, as it could in principal be possible for a change in effective diffusion length to be caused by a shift in the surface Fermi level pinning rather than by a change of positron trap density or type.

Our measurements tend to confirm the findings of Berwick et al. ${ }^{5}$ that within the space charge region adjacent to the metal/SI-GaAs contact the EL2 defects are only at most $\sim 0.1 \%$ ionized. The present theoretical model based on the work of McGregor et al. ${ }^{5}$ does not cater for this effect but only for a higher electric field space charge neutralization occurring around $10 \mathrm{kV} \mathrm{cm}^{-1}$ [as represented by the last factor in Eq. (7)]. Our finding is that to get agreement with the data, the first factor $N_{D}-N_{A}$ in Eq. (7) must also depend on the electric field to represent a low-field enhancement in the $\mathrm{EL}^{+}$capture cross section. The effective values of $N_{D}$ $-N_{A}$ seem to be in agreement with the Johnson model of enhanced $L$-band EL2 ${ }^{+}$capture produced by the onset of the Gunn effect at lower electric fields. ${ }^{39}$

The very "hard" saturation that occurs around electric field strengths $\sim 12 \mathrm{kV} \mathrm{cm}^{-1}$ may well be due to an even higher energy (electric field) threshold for enhanced electron capture that is not directly associated with transitions to the $L$ band. Alternatively it is equally likely that both the lowand high-field enhanced captures are manifestations of the same process. More sophisticated modelling of the processes occurring at the metal SI-GaAs interface together with more positron beam data will be required in gaining a better understanding of the mechanisms that give rise to the observed charge distributions under different reverse bias conditions. Furthermore, the sensitivity of using the positron as an electric field probe for the metal-semiconductor system could be improved upon in future studies of this sort by using a thinner metal overlayer thus allowing less positrons to annihilate in the metalization and a greater fraction to annihilate in the electric field region of interest. For example, in relation to the present $\mathrm{Au} / \mathrm{GaAs}$ systems, the fraction of positrons annihilating in the $\mathrm{Au}$ overlayer could be reduced from 0.54 to 0.18 if the Au film thickness were reduced to $500 \AA$.

\section{ACKNOWLEDGMENT}

The authors would like to thank the Research Grants Council of Hong Kong and the Croucher Foundation for grants made available for the development of the positron beam used in this work.

${ }^{1}$ R. B. Darling, J. Appl. Phys. 74, 4571 (1993).

${ }^{2}$ F. Dubecký, J. Darmo, J. Betko, Z. Mozolová, and P. G. Pelfer, Semicond. Sci. Technol. 9, 1654 (1994).

${ }^{3}$ S. P. Beaumont et al., Nucl. Instrum. Methods Phys. Res. A 326, 313 (1993).

${ }^{4}$ K. Berwick, M. R. Brozel, C. M. Buttar, M. Cowperthwaite, P. Sellin, and Y. Hou, Mater. Sci. Eng. B 28, 485 (1994).

${ }^{5}$ D. S. McGregor, R. A. Rojeski, G. F. Knoll, F. L. Terry, Jr., J. East, and Y. Eisen, J. Appl. Phys. 75, 7910 (1994).

${ }^{6}$ M. J. Puska and R. M. Nieminen, Rev. Mod. Phys. 66, 841 (1994).

${ }^{7}$ H. L. Au, C. C. Ling, B. K. Panda, T. C. Lee, C. D. Beling, and S. Fung, Phys. Rev. Lett. 73, 2732 (1994).

${ }^{8}$ C. D. Beling, R. I. Simpson, M. G. Stewart, Y. Y. Wang, S. Fung, C. H. 
Wai, and T. N. Sun, Phys. Status Solidi A 102, 537 (1987).

${ }^{9}$ R. I. Simpson, M. G. Stewart, C. D. Beling, and M. Charlton, J. Phys.: Condens. Matter 1, 7251 (1989).

${ }^{10}$ A. P. Mills, Jr. and L. Pfeiffer, Phys. Lett. A 63, 118 (1977).

${ }^{11}$ P. J. Schultz and K. G. Lynn, Rev. Mod. Phys. 60, 701 (1988).

${ }^{12}$ J. Mäkinen, C. Corbel, P. Hautojärvi, A. Vehanen, and D. Mathiot, Phys. Rev. B 42, 1750 (1990).

${ }^{13}$ Y. Y. Shan, H. L. Au, C. C. Ling, T. C. Lee, B. K. Panda, S. Fung, C. D. Beling, Y. Y. Wang, and M. M. Weng, Appl. Phys. A 59, 259 (1994).

${ }^{14}$ Y. Y. Shan, P. Asoka-Kumar, K. G. Lynn, S. Fung, and C. D. Beling, Phys. Rev. B 54, 1982 (1996).

${ }^{15}$ J. V. DiLorenzo and D. D. Khandelwal, GaAs FET Principles and Technology (Artech House, Dedham, 1982).

${ }^{16}$ T. P. Chen, Y. C. Liu, S. Fung, and C. D. Beling, J. Appl. Phys. 78, 4796 (1995).

${ }^{17}$ S. M. Sze, Physics of Semiconductor Devices, 2nd ed. (Wiley, New York, 1981).

${ }^{18}$ C. G. B. Garrett and W. E. Brattain, Phys. Rev. 99, 376 (1955).

${ }^{19}$ T. P. Chen, Y. C. Liu, S. Fung, and C. D. Beling, Solid State Commun. 94, 287 (1995).

${ }^{20}$ E. H. Rhoderick and R. H. Williams, Metal-Semiconductor Contacts, 2nd ed. (Clarendon, Oxford, 1988).

${ }^{21}$ V. Ya. Prinz and S. N. Rechkunov, Phys. Status Solidi B 118, 159 (1983).

${ }^{22}$ C. D. Beling, S. Fung, H. M. Weng, C. V. Reddy, S. W. Fan, Y. Y. Shan, and C. C. Ling, AIP Conf. Proc. 303, 462 (1992).

${ }^{23}$ A. Uedono, L. Wei, Y. Tabuki, H. Kondo, S. Tanigawa, K. Wada, and H. Nakanishi, Jpn. J. Appl. Phys. 30, L2002 (1991); A. Uedono, L. Wei, Y. Tabuki, H. Kondo, S. Tanigawa, K. Wada, and H. Nakanishi, Jpn. J. Appl. Phys. 30, L2002 (1991).
${ }^{24}$ H. E. Evans, P. C. Rice-Evans, D. L. Smith, G. A. Gledhill, and A. M. Moore, J. Phys.: Condens. Matter 4, 1859 (1992).

${ }^{25}$ P. C. Rice-Evans and D. L. Smith, Phys. Lett. A 141, 201 (1989).

${ }^{26}$ C. C. Ling, T. C. Lee, S. Fung, C. D. Beling, H. Weng, J. Xu, S. Sun, and R. Han, J. Phys.: Condens. Matter 6, 1133 (1994).

${ }^{27}$ A. van Veen, H. Schut, R. A. J. de Vries, R. A. Hakvoort, and M. R. Ijpma, AIP Conf. Proc. 218, 171 (1991).

${ }^{28}$ A. Vehanen, K. Saarinen, P. Hautojärvi, and H. Huomo, Phys. Rev. B 35, 4606 (1987).

${ }^{29}$ J. A. Baker, N. B. Chilton, K. O. Jensen, A. B. Walker, and P. G. Coleman, Appl. Phys. Lett. 59, 2962 (1991).

${ }^{30}$ C. C. Ling, Y. Y. Shan, B. K. Panda, S. Fleischer, C. D. Beling, and S. Fung, Appl. Phys. A 60, 545 (1995).

${ }^{31}$ G. Dlubek, O. Brümmer, F. Plazaola, and P. Hautojärvi, J. Phys. C 19, 331 (1986).

${ }^{32}$ A. P. Mills, Jr. and C. A. Murray, Appl. Phys. 21, 323 (1980).

${ }^{33}$ E. Soininen, J. Mäkinen, D. Beyer, and P. Hautojärvi, Phys. Rev. B 46, 13104 (1992).

${ }^{34}$ K. Saarinen, P. Hautojarvi, A. Vehanen, R. Krause, and G. Dlubek, Phys. Rev. B 39, 5287 (1989).

${ }^{35}$ H. L. Au, C. C. Ling, T. C. Lee, C. D. Beling, and S. Fung, Mater. Sci. Forum 105-111, 591 (1992).

${ }^{36}$ J. L. Lee, L. Wei, S. Tanigawa, H. Oigawa, and Y. Nannichi, Appl. Phys. Lett. 58, 1167 (1991).

${ }^{37}$ W. Z. Yu and B. S. Cao, Mater. Sci. Forum 105-111, 869 (1992).

${ }^{38}$ Y. L. Luo, T. P. Chen, S. Fung, C. D. Beling, Solid State Commun. (in press).

${ }^{39}$ D. A. Johnson, Ph.D. thesis, Arizona State University, 1989. 